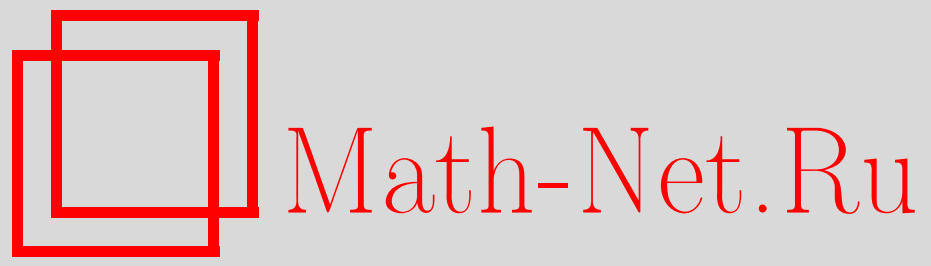

И. М. Щепочкина, Пять простых исключительных супералгебр Ли векторных полей, Функи. анализ и его прил., 1999, том 33, выпуск 3, 59-72

DOI: https://doi.org/10.4213/faa366

Использование Общероссийского математического портала MathNet.Ru подразумевает, что вы прочитали и согласны с пользовательским соглашением

http://www . mathnet.ru/rus/agreement

Параметры загрузки:

IP : 54.92 .164 .108

26 апреля 2023 г., 15:51:14

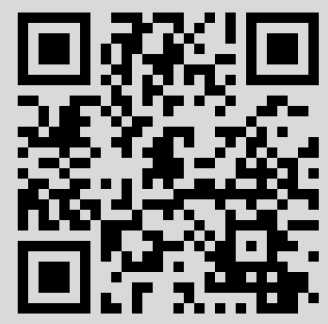


Функииональньй анализ и его приложения

1999, т. 33, вып. 3, с. 59-72

УДК 519.46

\title{
Пять простых исключительных супералгебр Ли векторных полей
}

\author{
(c) 1999. И. М. ЩЕПОЧКИНА
}

В. Кац [3] классифицировал простые конечномерные супералгебры Ли над $\mathbb{C}$. Он также предположил, что в классе бесконечномерных фильтрованных супералгебр Ли векторных полей полиномиального роста простыми являются лишь прямые аналоги четырех картановских серий $\mathfrak{v e c t}(n), \mathfrak{s v e c t}(n), \mathfrak{h}(2 n)$ и $\mathfrak{k}(2 n+1)$ всех, бездивергентных, гамильтоновых и контактных векторных полей соответственно.

Вскоре, однако, были найдены [6] и непрямые аналоги - четыре серии: $\mathfrak{m}$ и $\mathfrak{s m}$ - «нечетные» аналоги серии $\mathfrak{k}, \mathfrak{l} \mathfrak{e}$ и $\mathfrak{s l e}$ - «нечетные» аналоги серии $\mathfrak{h}$, а также некоторые их деформации $[4,5,7,8]$. Кроме того, были получены нестандартные реализации всех этих серий [1], т. е. другие фильтрованные супералгебры Ли, изоморфные указанным выше как абстрактные супералгебры.

Наконец, в $[10,11]$ приведены краткие описания четырех простых исключительных супералгебр Ли векторных полей, а препринт [9] посвящен изучению одной из них. Целью настоящей работы является более детальное описание этих алгебр и определение наиболее замечательной пятой простой исключительной супералгебры. Связанные с ней конструкции для струнных супералгебр Ли см. в [2].

Автор выражает глубокую благодарность Д. Лейтесу за постановку задачи и внимание к работе, Г. Посту и П. Грозману за неоценимую помощь в компьютерных вычислениях и университетам Стокгольма и Твенте за гостеприимство.

\section{$\S 1$. Основные обозначения и предварительные сведения}

Всюду в дальнейшем основное поле - поле $\mathbb{C}$.

1.1. Пусть $x=\left(u_{1}, \ldots, u_{n}, \theta_{1}, \ldots, \theta_{m}\right)$, где $u_{i}$ - четные переменные, а $\theta_{j}$ нечетные. Через $\mathfrak{v e c t}(n \mid m)$ мы обозначаем супералгебру Ли $\mathfrak{d e r} \mathbb{C}[x]$ всех векторных полей от $x$ с полиномиальными коэффициентами. Естественная $\mathbb{Z}$-градуировка в многочленах $\operatorname{deg} u_{i}=\operatorname{deg} \theta_{j}=1$ порождает $\mathbb{Z}$-градуировку в $\mathfrak{v e c t}(n \mid m)$.

Дивергенцией векторного поля $D=\sum_{i} f_{i} \partial / \partial u_{i}+\sum_{j} g_{j} \partial / \partial \theta_{j}$ называется функция

$$
\operatorname{div} D=\sum_{i} \frac{\partial f_{i}}{\partial u_{i}}+\sum_{j}(-1)^{p\left(g_{j}\right)} \frac{\partial g_{i}}{\partial \theta_{j}} .
$$

Супералгебра Ли специальных, или бездивергентных векторных полей $\mathfrak{s v e c t}(n \mid m)=\{D \in \operatorname{vect}(n \mid m): \operatorname{div} D=0\}$ состоит из полей, сохраняющих форму объема $\operatorname{vol}_{x}$ с постоянными коэффициентами в координатах $x$.

* Работа выполнена при частичной поддержке Международной ассоциации INTAS (грант 96-0538). 
Пусть $u=\left(t, p_{1}, \ldots, p_{n}, q_{1}, \ldots, q_{n}\right), \theta=\left(\xi_{1}, \ldots, \xi_{r}, \eta_{1}, \ldots, \eta_{r}\right)$ и

$$
\alpha_{1}=d t+\sum_{1 \leqslant i \leqslant n}\left(p_{i} d q_{i}-q_{i} d p_{i}\right)+\sum_{1 \leqslant j \leqslant r}\left(\xi_{j} d \eta_{j}+\eta_{j} d \xi_{j}\right), \quad \omega_{0}=d \alpha_{1} .
$$

Супералгебра Ли, сохраняющая уравнение Пфаффа $\alpha_{1}=0$,

$$
\mathfrak{k}(2 n+1 \mid 2 r)=\left\{D \in \operatorname{vect}(2 n+1 \mid 2 r): L_{D} \alpha_{1}=f_{D} \alpha_{1}, f_{D} \in \mathbb{C}[t, p, q, \xi, \eta]\right\},
$$

называется контактной супералгеброй Ли, а супералгебра Ли, сохраняющая саму форму $\alpha_{1}$,

$$
\mathfrak{p o}(2 n \mid 2 r)=\left\{D \in \mathfrak{k}(2 n+1 \mid 2 r): L_{D} \alpha_{1}=0\right\}
$$

называется пуассоновской. (Отметим, что, вообще говоря, число нечетных переменных $\theta_{i}$ не обязано быть четным. Однако в интересующих нас примерах оно четно; поэтому мы приводим формулы лишь для этого случая.)

«Нечетные» аналоги этих супералгебр Ли получаются, если

$$
u=q=\left(q_{1}, \ldots, q_{n}\right), \quad \theta=\left(\xi_{1}, \ldots, \xi_{n} ; \tau\right)
$$

и

$$
\alpha_{0}=d \tau+\sum_{i}\left(\xi_{i} d q_{i}+q_{i} d \xi_{i}\right), \quad \omega_{1}=d \alpha_{0} .
$$

Супералгебра Ли, сохраняющая уравнение Пфаффа $\alpha_{0}=0$,

$$
\mathfrak{m}(n)=\left\{D \in \mathfrak{v e c t}(n \mid n+1): L_{D} \alpha_{0}=f_{D} \alpha_{0}\right\}, \quad \text { где } f_{D} \in \mathbb{C}[q, \xi, \tau],
$$

называется нечетной контактной, а супералгебра Ли, сохраняющая $\alpha_{0}$,

$$
\mathfrak{b}(n)=\left\{D \in \mathfrak{m}(n): L_{D} \alpha_{0}=0\right\},
$$

называется бютановской.

В отличие от четного случая, супералгебры Ли $\mathfrak{m}(n)$ и $\mathfrak{b}(n)$ не сохраняют форму объема. Поэтому определены их специальные подалгебры

$$
\mathfrak{s m}(n)=\{D \in \mathfrak{m}(n): \operatorname{div} D=0\}, \quad \mathfrak{s b}(n)=\{D \in \mathfrak{b}(n): \operatorname{div} D=0\} .
$$

Супералгебры Ли ḱ, $\mathfrak{m}$ и их подалгебры удобно описывать с помощью производящих функций. Пусть $E=\sum_{i} y_{i} \partial / \partial y_{i}$, где $y$ - это все координаты, кроме $t$ (в случае $\mathfrak{k}$ ) или $\tau$ (в случае $\mathfrak{m})$, a $\nabla(f)=2 f-E(f)$. Тогда в случае формы $\alpha_{1}$ для $f \in \mathbb{C}[t, p, q, \xi, \eta]$ положим

где

$$
K_{f}=\nabla(f) \frac{\partial}{\partial t}+H_{f}+\frac{\partial f}{\partial t} E,
$$

$$
H_{f}=\sum_{i \leqslant n}\left(\frac{\partial f}{\partial p_{i}} \frac{\partial}{\partial q_{i}}-\frac{\partial f}{\partial q_{i}} \frac{\partial}{\partial p_{i}}\right)-(-1)^{p(f)} \sum_{j \leqslant k}\left(\frac{\partial f}{\partial \xi_{j}} \frac{\partial}{\partial \eta_{j}}+\frac{\partial f}{\partial \eta_{j}} \frac{\partial}{\partial \xi_{j}}\right) .
$$

Если $f \in \mathbb{C}[p, q, \xi, \eta]$, т. е. $f$ не зависит от $t$, то $H_{f}$ - гамильтоново векторное поле, сохраняющее форму $\omega_{0}$.

В случае формы $\alpha_{0}$ для $f \in \mathbb{C}[q, \xi, \tau]$ положим

где

$$
M_{f}=\nabla(f) \frac{\partial}{\partial \tau}+\operatorname{Le}_{f}-(-1)^{p(f)} \frac{\partial f}{\partial \tau} E,
$$

$$
\operatorname{Le}_{f}=\sum_{i \leqslant n}\left(\frac{\partial f}{\partial q_{i}} \frac{\partial}{\partial \xi_{i}}+(-1)^{p(f)} \frac{\partial f}{\partial \xi_{i}} \frac{\partial}{\partial q_{i}}\right)
$$


Отметим, что $p\left(\operatorname{Le}_{f}\right)=p\left(M_{f}\right)=p(f)+\overline{1}$, и если $f \in \mathbb{C}[q, \xi]$, т. е. $f$ не зависит от $\tau$, то Lе $f$ - поле, сохраняющее $\omega_{1}$.

Нетрудно проверить, что

$$
L_{K_{f}}\left(\alpha_{1}\right)=2(\partial f / \partial t) \alpha_{1}, \quad L_{M_{f}}\left(\alpha_{0}\right)=-(-1)^{p(f)} 2(\partial f / \partial \tau) \alpha_{0} .
$$

Поэтому $K_{f} \in \mathfrak{k}(2 n+1 \mid 2 r)$, a $M_{f} \in \mathfrak{m}(n)$.

Коммутаторам векторных полей $\left[K_{f}, K_{g}\right]$ и $\left[M_{f}, M_{g}\right]$ соответствуют контактные скобки производящих функций: $\left[K_{f}, K_{g}\right]=K_{\{f, g\}_{\mathrm{k.b}} .},\left[M_{f}, M_{g}\right]=$ $M_{\{f, g\}_{\text {m.b. }}, \text { где }}$

$$
\begin{aligned}
\{f, g\}_{\text {k.b. }} & =\nabla(f) \frac{\partial g}{\partial t}-\frac{\partial f}{\partial t} \nabla(g)+\{f, g\}_{\text {P.b. }} \\
\{f, g\}_{\text {m.b. }} & =\nabla(f) \frac{\partial g}{\partial \tau}+(-1)^{p(f)} \frac{\partial f}{\partial \tau} \nabla(g)+\{f, g\}_{\text {B.b. }},
\end{aligned}
$$

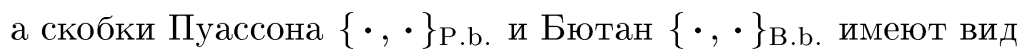

$$
\begin{aligned}
\{f, g\}_{\text {P.b. }} & =\sum_{i \leqslant n}\left(\frac{\partial f}{\partial p_{i}} \frac{\partial g}{\partial q_{i}}-\frac{\partial f}{\partial q_{i}} \frac{\partial g}{\partial p_{i}}\right)-(-1)^{p(f)} \sum_{j \leqslant r}\left(\frac{\partial f}{\partial \xi_{j}} \frac{\partial g}{\partial \eta_{j}}+\frac{\partial f}{\partial \eta_{j}} \frac{\partial g}{\partial \xi_{j}}\right), \\
\{f, g\}_{\text {B.b. }} & =\sum_{i \leqslant n}\left(\frac{\partial f}{\partial q_{i}} \frac{\partial g}{\partial \xi_{i}}+(-1)^{p(f)} \frac{\partial f}{\partial \xi_{i}} \frac{\partial g}{\partial q_{i}}\right) .
\end{aligned}
$$

На языке производящих функций супералгебра $\mathfrak{b}(n)$ выделяется в $\mathfrak{m}(n)$ уравнением $\partial f / \partial \tau=0$, а супералгебра $\mathfrak{s m}(n)-$ уравнением $\operatorname{div} M_{f}=0$. Оказывается, что любая линейная комбинация этих уравнений задает некоторую (почти всегда простую) подалгебру в $\mathfrak{m}(n)$. Положим

$$
\mathfrak{b}_{a, b}(n)=\left\{M_{f} \in \mathfrak{m}(n): a \operatorname{div} M_{f}=(-1)^{p(f)} 2(a-b n) \partial f / \partial \tau\right\} .
$$

Заметим, что супералгебры Ли ро и $\mathfrak{b}$ содержат одномерные центры, факторы по которым образуют гамильтоновы (четную и нечетную соответственно) супералгебры Ли $\mathfrak{h}$ и $\mathfrak{l}$,

$$
\mathfrak{h}(2 n \mid 2 m) \cong \operatorname{Span}\left(H_{f}: f \in \mathbb{C}[p, q, \xi, \eta]\right), \quad \mathfrak{l}(n) \cong \operatorname{Span}\left(\operatorname{Le}_{f}: f \in \mathbb{C}[q, \xi]\right) .
$$

Специальные подалгебры $\mathfrak{s l e}(n)=\mathfrak{l}(n) \cap \mathfrak{s v e c t}(n \mid n)$ и $\mathfrak{s b}(n)=\mathfrak{b}(n) \cap \mathfrak{s m}(n)$ выделяются в производящих функциях уравнением $\Delta(f)=0$, где $\Delta=\sum_{i \leqslant n} \partial^{2} / \partial q_{i} \partial \xi_{i}$ - нечетный аналог оператора Лапласа.

Супералгебры Ли $\mathfrak{s l e}(n)$ и $\mathfrak{s b}(n)$ содержат идеалы $\mathfrak{s l e}^{\circ}(n)$ и $\mathfrak{s b}^{\circ}(n)$ коразмерности 1 , определяемые точными последовательностями

$$
\begin{aligned}
& 0 \longrightarrow \mathfrak{s l e}^{\circ}(n) \longrightarrow \mathfrak{s l e}(n) \longrightarrow \mathbb{C} \cdot \operatorname{Le}_{\xi_{1} \ldots \xi_{n}} \longrightarrow 0, \\
& 0 \longrightarrow \mathfrak{s b}^{\circ}(n) \longrightarrow \mathfrak{s b}(n) \longrightarrow \mathbb{C} \cdot M_{\xi_{1} \ldots \xi_{n}} \longrightarrow 0 .
\end{aligned}
$$

В терминах производящих функций $\mathbb{Z}$-градуировка gr в супералгебрах $\mathfrak{k}$ и $\mathfrak{m}$ определяется условиями $\operatorname{deg} y_{i}=1, \operatorname{deg} t=\operatorname{deg} \tau=2, \operatorname{gr}(f)=\operatorname{deg} f-2$.

1.2. Производящие функции для $\mathfrak{s b}^{\circ}(n)$ составляют образ нечетного оператора Лапласа $\Delta$. Пусть $f \in \mathfrak{s b}^{\circ}(n)$ - произвольная однородная по $q$ и $\xi$ гармоническая функция, а $\Phi=\sum q_{i} \xi_{i}$. Тогда

$$
\Delta(\Phi f)=(\Delta \Phi) f-\Phi \Delta f-\{\Phi, f\}=\left(n+\operatorname{deg}_{q} f-\operatorname{deg}_{\xi} f\right) f .
$$


Определим правый обратный к оператору $\Delta$ по формуле

$$
\Delta^{-1} f=\frac{1}{\nu(f)}(\Phi f), \quad \text { где } \nu(f)=n+\operatorname{deg}_{q} f-\operatorname{deg}_{\xi} f .
$$

Если считать, что мы реализуем супералгебру $\mathfrak{l}(n)$ производящими функциями $f, f(0)=0$, то оператор $\Delta^{-1}$ будет определен и на $\mathfrak{s l e} \mathfrak{e}^{\circ}(n)$. В обоих случаях будет выполнено равенство $\Delta\left(\Delta^{-1} f\right)=f$.

1.3. В отличие от алгебр Ли, все описанные супералгебры Ли допускают несколько реализаций в виде векторных полей. Нас будет интересовать одна нестандартная реализация супералгебр Ли $\mathfrak{b}_{a, b}(n), \mathfrak{l} \mathfrak{e}(n)$ и $\mathfrak{s l e}^{\circ}(n)$.

Реализуем $\mathfrak{g}=\mathfrak{b}_{a, b}(n)$ производящими функциями от $\tau, q_{1}, \ldots, q_{n}, \xi_{1}, \ldots, \xi_{n}$, а $\mathfrak{g}=\mathfrak{l} \mathfrak{e}(n)$ или $\mathfrak{s l e}{ }^{\circ}(n)$ производящими функциями от $q_{1}, \ldots, q_{n}, \xi_{1}, \ldots, \xi_{n}$ и введем новую $\mathbb{Z}$-градуировку gr $f=\operatorname{deg} f-1$, где $\operatorname{deg} \tau=\operatorname{deg} q_{i}=1, \operatorname{deg} \xi_{i}=0$. Результат этой переградуировки обозначим через $R(\mathfrak{g})$. Тогда $R(\mathfrak{g})=\bigoplus_{i \geqslant-1}(R(\mathfrak{g}))_{i}$ и, значит, $R(\mathfrak{g})$ реализуется как подалгебра супералгебры Ли $\mathfrak{v e c t}(N \mid M)$ с некоторыми новыми $N$ и $M$. Мы используем для $R(\mathfrak{g})$ обозначения $\mathfrak{b}_{a, b}(n ; n)$, $\mathfrak{l}(n ; n)$ и $\mathfrak{s l e}^{\circ}(n ; n)$ соответственно.

Для $R(\mathfrak{g})=\mathfrak{l e}(n ; n)$ имеем $(R(\mathfrak{g}))_{-1}=(\Pi(\Lambda(n)) / \mathbb{C} \cdot 1)$, где П - оператор изменения четности, $(R(\mathfrak{g}))_{0}=\mathfrak{v e c t}(0 \mid n)$ и действие $(R(\mathfrak{g}))_{0}$ на $(R(\mathfrak{g}))_{-1}-$ это естественное действие векторных полей на функции.

Для $R(\mathfrak{g})=\mathfrak{s l e}^{\circ}(n ; n)$ имеем $(R(\mathfrak{g}))_{-1}=(\Pi(\tilde{\Lambda}(n)) / \mathbb{C} \cdot 1)$, где $\tilde{\Lambda}(n)=\{f \in$ $\Lambda(n) \mid \int f$ vol $\left.=0\right\}$, a $(R(\mathfrak{g}))_{0}=\mathfrak{s v e c t}(0 \mid n)$. При этом естественное действие специальных векторных полей на функциях сохраняет невырожденную суперкососимметрическую форму $\omega, \omega(f, g)=\int f g$ vol.

Наконец, для $R(\mathfrak{g})=\mathfrak{b}_{a, b}(n ; n)$ имеем $(R(\mathfrak{g}))_{-1}=\Pi(\Lambda(n)),(R(\mathfrak{g}))_{0}=\mathfrak{v e c t}(0 \mid n)$. Однако действие $(R(\mathfrak{g}))_{0}$ на $(R(\mathfrak{g}))_{-1}$ имеет вид

$$
[D, f]=D f+\lambda \operatorname{div} D, \quad \text { где } f \in \Lambda(n), D \in \mathfrak{v e c t}(0 \mid n), \lambda=\frac{2 a}{n(a-b)},
$$

т. е. как $(R(\mathfrak{g}))_{0}$-модуль пространство $(R(\mathfrak{g}))_{-1}$ является пространством $\lambda$-плотностей. Поэтому мы используем также обозначения $(R(\mathfrak{g}))_{-1}=\Pi\left(\Lambda_{\lambda}(n)\right)$ и $R(\mathfrak{g})=\mathfrak{b}_{\lambda}(n ; n)$.

1.4. Пусть $\mathfrak{g}=\mathfrak{s l e}^{\circ}(3)$. В стандартной $\mathbb{Z}$-градуировке $\operatorname{dim} \mathfrak{g}_{-1}=(3,3), \mathfrak{g}_{0} \cong$ $\mathfrak{s p e}(3)$. Для переградуированной супералгебры Ли $R(\mathfrak{g})=\mathfrak{s l e}^{\circ}(3 ; 3)$ имеем $\operatorname{dim}(R(\mathfrak{g}))_{-1}=(3,3),(R(\mathfrak{g}))_{0}=\mathfrak{s v e c t}(0 \mid 3) \cong \mathfrak{s p e}(3)$. Следовательно, переградуировка $R$ определяет нетривиальный автоморфизм супералгебры Ли $\mathfrak{s l e} \mathfrak{e}^{\circ}(3)$. $\mathrm{B}$ терминах производящих функций оператор $R$ задается следующими формулами:

1) если $\operatorname{deg}_{\xi}(f)=0$, то $R(f)=\Delta\left(f \xi_{1} \xi_{2} \xi_{3}\right)$;

2) если $\operatorname{deg}_{\xi}(f)=1$, то $R(f)=f$;

3) если $\operatorname{deg}_{\xi}(f)=2$, то $R(f)=\partial^{3}\left(\Delta^{-1} f\right) / \partial \xi_{1} \partial \xi_{2} \partial \xi_{3}$.

При этом $R^{2}=\operatorname{sign}$, где $\operatorname{sign}(f)=(-1)^{p(f)+1} f$.

Супералгебра Ли $\mathfrak{g}=\mathfrak{s l e} \mathfrak{e}^{\circ}(3)$ обладает $(0 \mid 2)$-мерным центральным расширением $\mathfrak{e s l e} \mathfrak{e}^{\circ}(3)$ : элемент $M_{1}$ степени -2 относительно стандартной $\mathbb{Z}$-градуировки в $\mathfrak{s l e}^{\circ}(3)$ расширяет $\mathfrak{s l e}^{\circ}(3)$ до $\mathfrak{s b}^{\circ}(3)$, а элемент $z$ степени -2 относительно градуировки в $\mathfrak{s l e}^{\circ}(3 ; 3)$ задает расширение с помощью инвариантной формы $\omega$. 
Переградуировка $R$ переставляет эти центральные элементы и задает нетривиальный автоморфизм супералгебры Ли $\mathfrak{e s l e}^{\circ}(3)$.

1.5. Основной конструкцией, используемой при построении исключительных супералгебр Ли векторных полей, является конструкция картановского продолжения [12] и ее обобщения [10]. Пусть $\mathfrak{g}$ - супералгебра Ли, $V$ есть $(n \mid m)$-мерный $\mathfrak{g}$-модуль и $S^{i}$ - оператор $i$-й суперсимметрической степени. Положим $\mathfrak{g}_{-1}=V, \mathfrak{g}_{0}=\mathfrak{g}$ и определим $i$-е картановское продолжение для $i>0$ KaK

$$
\begin{aligned}
\mathfrak{g}_{i} & =\left\{X \in \operatorname{Hom}\left(\mathfrak{g}_{-1}, \mathfrak{g}_{i-1}\right): X\left(w_{0}\right)\left(w_{1}, \ldots, w_{i}\right)\right. \\
& \left.=(-1)^{p\left(w_{0}\right) p\left(w_{1}\right)} X\left(w_{1}\right)\left(w_{0}, \ldots, w_{i}\right) \text { для любых } w_{0}, w_{1} \in \mathfrak{g}_{-1}\right\} \\
& =\left(S^{i}\left(\mathfrak{g}_{-1}\right)^{*} \otimes \mathfrak{g}_{0}\right) \cap\left(S^{i+1}\left(\mathfrak{g}_{-1}\right)^{*} \otimes \mathfrak{g}_{-1}\right) .
\end{aligned}
$$

(В последнем выражении мы рассматриваем $\mathfrak{g}_{0}$ как подпространство в $\mathfrak{g}_{-1}^{*} \otimes$ $\mathfrak{g}_{-1}$; поэтому пересечение корректно определено.)

Алгебра $\left(\mathfrak{g}_{-1}, \mathfrak{g}_{0}\right)_{*}=\bigoplus_{i \geqslant-1} \mathfrak{g}_{i}$ называется картановским продолжением пары $(V, \mathfrak{g})$. Ясно, что если $\mathfrak{g}_{0}$-модуль $\mathfrak{g}_{-1}$ точен, то $\left(\mathfrak{g}_{-1}, \mathfrak{g}_{0}\right)_{*} \subset \mathfrak{v e c t}(n \mid m)=$ $\operatorname{der} \mathbb{C}[x]$,

$$
\mathfrak{g}_{i}=\left\{D \in \operatorname{vect}(n \mid m): \operatorname{deg} D=i,[D, X] \in \mathfrak{g}_{i-1} \text { для всех } X \in \mathfrak{g}_{-1}\right\}
$$

и $\left(\mathfrak{g}_{-1}, \mathfrak{g}_{0}\right)_{*}$ является максимальной подалгеброй в $\mathfrak{v e c t}(n \mid m)$ с данной неположительной частью. При этом $D=\sum_{i} f_{i} \partial / \partial x_{i} \in\left(\mathfrak{g}_{-1}, \mathfrak{g}_{0}\right)_{*}$ тогда и только тогда, когда матрица $P(D)=\left(P_{i, j}\right)$, где $P_{i, j}=(-1)^{p(D) p\left(x_{j}\right)} \partial f_{i} / \partial x_{j}$ принадлежит $\mathfrak{g}_{0} \otimes \mathbb{C}[x]$. Другими словами, картановское продолжение всегда выделяется системой линейных дифференциальных уравнений в частных производных.

Нам потребуются следующие равенства:

$$
\begin{aligned}
((\Pi(\Lambda(n)) / \mathbb{C} \cdot 1), \mathfrak{v e c t}(0 \mid n))_{*} & =\mathfrak{l} \mathfrak{e}(n ; n), \\
((\Pi(\tilde{\Lambda}(n)) / \mathbb{C} \cdot 1), \mathfrak{s v e c t}(0 \mid n))_{*} & =\mathfrak{s l e}^{\circ}(n ; n), \\
\left(\Pi\left(\Lambda_{\lambda}(n)\right), \mathfrak{v e c t}(0 \mid n)\right)_{*} & =\mathfrak{b}_{\lambda}(n ; n) .
\end{aligned}
$$

1.6. Опишем теперь конструкцию, обобщающую картановское продолжение. Пусть $\mathfrak{g}_{-}=\bigoplus_{-d \leqslant i \leqslant-1} \mathfrak{g}_{i}$ есть нильпотентная $\mathbb{Z}$-градуированная супералгебра Ли и $\mathfrak{g}_{0} \subset \mathfrak{d e r}_{0} \mathfrak{g}_{-}$- некоторая подалгебра супералгебры Ли дифференцирований супералгебры $\mathfrak{g}_{-}$, сохраняющих $\mathbb{Z}_{-}$ррадуировку. Для $i>0$ определим $i$-е продолжение пары $\left(\mathfrak{g}_{-}, \mathfrak{g}_{0}\right)$ как

$$
\mathfrak{g}_{i}=\left(\left(S^{\cdot}\left(\mathfrak{g}_{-}\right)^{*} \otimes \mathfrak{g}_{0}\right) \cap\left(S^{\cdot}\left(\mathfrak{g}_{-}\right)^{*} \otimes \mathfrak{g}_{-}\right)\right)_{i},
$$

где индекс $i$ в правой части означает компоненту степени $i$, а $S \cdot$ есть сумма всех $S^{j}$. Положим $\left(\mathfrak{g}_{-}, \mathfrak{g}_{0}\right)_{*}=\bigoplus_{i \geqslant-d} \mathfrak{g}_{i}$.

Нас будут особо интересовать два случая. Пусть $W$ есть $(2 n \mid m)$-мерное суперпространство, снабженное четной невырожденной суперкососимметрической билинейной формой $\omega$, а $\mathbb{C} z$ есть $(1 \mid 0)$-мерное пространство с базисным элементом $z$. На $\mathfrak{g}_{-}=W \oplus \mathbb{C} z$ определим скобку: $z-$ центральный элемент, $\mathbf{a}[v, w]=\omega(v, w) z$ для любых $v, w \in W$. Тогда $\mathfrak{g}_{-}$превращается в су-

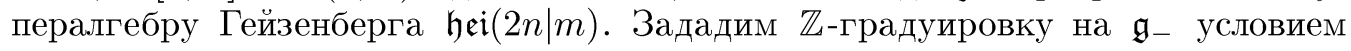


$\mathfrak{g}_{-1}=W, \mathfrak{g}_{-2}=\mathbb{C} z$. Тогда $\mathfrak{d e r}_{0} \mathfrak{g}_{-}=\mathfrak{c}(\mathfrak{o s p}(m \mid 2 n))$, где $\mathfrak{c}(\mathfrak{g})$ означает тривиальное центральное расширение, а $(\mathfrak{h e i}(2 n \mid m), \mathfrak{c}(\mathfrak{o s p}(m \mid 2 n)))_{*}=\mathfrak{k}(2 n+1 \mid m)$. Нетрудно проверить, что если $\mathfrak{g}_{0} \subset \mathfrak{c}(\mathfrak{o s p}(m \mid 2 n))$, то продолжение $\left(\mathfrak{h e i}(2 n \mid m), \mathfrak{g}_{0}\right)_{*}$ содержится в $\mathfrak{k}(2 n+1 \mid m)$ и является максимальной подалгеброй с данной неположительной частью.

Аналогичная процедура для $(n \mid n)$-мерного суперпространства $W$, снабженного нечетной невырожденной суперкососимметрической билинейной формой $\omega$, приводит к антискобочной супералгебре $\mathfrak{g}_{-}=\mathfrak{a} \mathfrak{b}(n), \mathfrak{d e r}_{0} \mathfrak{g}_{-}=\mathfrak{c}(\mathfrak{p e}(n))$ и $(\mathfrak{a} \mathfrak{b}(n), \mathfrak{c}(\mathfrak{p e}(n)))_{*}=\mathfrak{m}(n)$. Если $\mathfrak{g}_{0} \subset \mathfrak{c}(\mathfrak{p e}(n))$, то продолжение $\left(\mathfrak{a} \mathfrak{b}(n), \mathfrak{g}_{0}\right)_{*}$ содержится в $\mathfrak{m}(n)$ и является максимальной подалгеброй с данной неположительной частью.

Если $\mathfrak{g}_{-}$есть $\mathfrak{h e i}(2 n \mid m)$ или $\mathfrak{a} \mathfrak{b}(n)$, мы вместо $\left(\mathfrak{g}_{-}, \mathfrak{g}_{0}\right)_{*}$ используем символ $\left(\mathfrak{g}_{-}, \mathfrak{g}_{0}\right)_{*}^{\mathfrak{m} \mathfrak{k}}$, подчеркивая, что само продолжение является подалгеброй в $\mathfrak{k}$ или $\mathfrak{m}$.

1.7. Наконец, последняя конструкция, которую мы используем, - это конструкция картановского продолжения с элементов степени $\leqslant 1$.

Пусть $\mathfrak{g}=\bigoplus_{i \geqslant-d} \mathfrak{g}_{i}$ есть $\mathbb{Z}$-градуированная супералгебра Ли, такая, что $\mathfrak{g}_{-1}$ порождает всю $\mathfrak{g}_{-}$. Возьмем $\mathfrak{g}_{0}$-подмодуль $\mathfrak{h}_{1}$ в $\mathfrak{g}_{1}$. Определим второе продолжение пары $\left(\bigoplus_{i \leqslant 0} \mathfrak{g}_{i}, \mathfrak{h}_{1}\right)$ как $\mathfrak{h}_{2}=\left\{D \in \mathfrak{g}_{2}:\left[D, \mathfrak{g}_{-1}\right] \subset \mathfrak{h}_{1}\right\}$. Члены $\mathfrak{h}_{i}$ для $i>2$ определим аналогично: $\mathfrak{h}_{i}=\left\{D \in \mathfrak{g}_{i}:\left[D, \mathfrak{g}_{-1}\right] \subset \mathfrak{h}_{i-1}\right\}$. Положим $\mathfrak{h}_{i}=\mathfrak{g}_{i}$ для $i \leqslant 0$ и $\mathfrak{h}_{*}=\bigoplus \mathfrak{h}_{i}$. Нетрудно проверить, что, во-первых, $\mathfrak{h}_{*}$ является подсупералгеброй Ли в $\mathfrak{g}$ и, во-вторых, она максимальна среди всех подалгебр с заданной суммой компонент степени не выше 1.

\section{§2. Исключительная супералгебра Ли vas(4|4)}

Пусть $\omega$ есть нечетная невырожденная суперкососимметрическая билинейная форма на $(n \mid n)$-мерном суперпространстве $V$ с матрицей $\left(\begin{array}{cc}0 & 1_{n} \\ 1_{n} & 0\end{array}\right)$. Линейные преобразования, сохраняющие $\omega$, образуют супералгебру Ли $\mathfrak{p e}(n)$ и задаются (в действии на строки) матрицами вида $\left(\begin{array}{cc}a & b \\ c & -a^{t}\end{array}\right)$, где $b=b^{t}, c=-c^{t}$. Супералгебра Ли $\mathfrak{s p e}(n)$ выделяется условием tr $a=0$. Полагая

$$
\operatorname{deg}\left(\left(\begin{array}{ll}
0 & 0 \\
c & 0
\end{array}\right)\right)=-1, \quad \operatorname{deg}\left(\left(\begin{array}{cc}
a & 0 \\
0 & -a^{t}
\end{array}\right)\right)=0, \quad \operatorname{deg}\left(\left(\begin{array}{ll}
0 & b \\
0 & 0
\end{array}\right)\right)=1,
$$

мы задаем $\mathbb{Z}$-градуировку на $\mathfrak{p e}(n)$. Известно [3], что $\mathfrak{s p e}(n)=\mathfrak{p e}(n) \cap \mathfrak{s l}(n \mid n)$ является простой супералгеброй Ли при $n \geqslant 3$.

А. Н. Сергеев доказал, что лишь в одном случае, а именно при $n=4$, супералгебра Ли $\mathfrak{s p e}(n)$ обладает нетривиальным центральным расширением, которое мы обозначаем as. Представим произвольный элемент $A \in \mathfrak{a s}$ в виде пары $A=x+d z$, где $x \in \mathfrak{s p e}(4), d \in \mathbb{C}$, a $z$ - центральный элемент.

Супералгебра Ли as может быть описана с помощью спинорного представления. Пусть $\mathfrak{p o}(0 \mid 6)$ есть супералгебра Ли функций $\Lambda(\xi, \eta)$ от нечетных переменных $\xi_{1}, \xi_{2}, \xi_{3}, \eta_{1}, \eta_{2}, \eta_{3}$ со скобкой Пуассона, а $\mathfrak{h}(0 \mid 6)=\operatorname{Span}\left(H_{f}: f \in\right.$ $\Lambda(\xi, \eta))$. В естественной $\mathbb{Z}$-градуировке $\mathfrak{h}(0 \mid 6)=\bigoplus_{i=-1}^{4} \mathfrak{h}(0 \mid 6)_{i}$ нулевая компонента $\mathfrak{h}(0 \mid 6)_{0}$ равна $\mathfrak{o}(6) \cong \mathfrak{s l}(4) \cong \mathfrak{s p e}(4)_{0}$, а $(-1)$-е компоненты $\mathfrak{h}(0 \mid 6)_{-1}$ и $\mathfrak{s p e}(4)_{-1}$ являются изоморфными шестимерными модулями. В силу универсальности картановского продолжения $\mathfrak{s p e}(4)_{1}$ вкладывается в $\mathfrak{h}(0 \mid 6)_{1}$. Расширение 
Сергеева as является результатом ограничения на $\mathfrak{s p e}(4) \subset \mathfrak{h}(0 \mid 6)$ коцикла, превращающего $\mathfrak{h}(0 \mid 6)$ в $\mathfrak{p o}(0 \mid 6)$. Ясно, что $\operatorname{deg} z=-2$ относительно градуировки (2.1).

Квантование деформирует $\mathfrak{p o}(0 \mid 6)$ в $\mathfrak{g l}(\Lambda(\xi))$, а сквозное отображение

$$
T_{\lambda}: \mathfrak{a s} \rightarrow \mathfrak{p o}(0 \mid 6) \rightarrow \mathfrak{g l}(\Lambda(\xi))
$$

задает семейство представлений $\operatorname{spin}_{\lambda}$ супералгебры Ли as в (4|4)-мерном пространстве. Точные формулы для $T_{\lambda}$ таковы:

$$
T_{\lambda}:\left(\begin{array}{cc}
a & b \\
c & -a^{t}
\end{array}\right)+d z \longmapsto\left(\begin{array}{cc}
a & b-\lambda \tilde{c} \\
c & -a^{t}
\end{array}\right)+\lambda d \cdot 1_{4 \mid 4},
$$

где $1_{4 \mid 4}$ есть единичная матрица, а для кососимметрической матрицы $c_{i j}=$ $E_{i j}-E_{j i}$ мы полагаем $\tilde{c}_{i j}=c_{k l}$ для четной подстановки $(1234) \mapsto(i j k l)$. Ясно, что все $T_{\lambda}$ являются неприводимыми.

Теорема 2.1.1) Для всех $\lambda \neq 0$ картановское продолжение $\mathfrak{f}^{\lambda}=\left(\operatorname{spin}_{\lambda}, \mathfrak{a s}\right)_{*}$ является бесконечномерной и простой супералгеброй.

2) $\mathfrak{f}^{\lambda} \cong \mathfrak{f}^{\mu}$, если $\lambda \mu \neq 0$.

ДокАЗАТЕльство. Формула (2.2) определяет естественное вложение $\mathfrak{f}^{\lambda}$ в $\mathfrak{g}=$ $\mathfrak{v e c t}(4 \mid 4)$, такое, что $\mathfrak{f}_{-1}^{\lambda}=\mathfrak{g}_{-1}$. Пусть $q_{1}, q_{2}, q_{3}, q_{4}, \xi_{1}, \xi_{2}, \xi_{3}, \xi_{4}$ - координаты, в функциях от которых действует $\mathfrak{g}$. Тогда для любого $k \geqslant 0$ элемент $q_{i}^{k+1} \partial \xi_{i}$ принадлежит $\mathfrak{f}_{k}^{\lambda}$ и, значит, $\operatorname{dim} \mathfrak{f}^{\lambda}=\infty$.

Предположим, что $\mathfrak{f}^{\lambda}$ не проста и $\mathfrak{i}=\bigoplus_{k \geqslant-1} \mathfrak{i}_{k}$ является нетривиальным идеалом в $\mathfrak{f}^{\lambda}$. Пусть $x \in \mathfrak{i}_{k}, x \neq 0$. Тогда $\left(\operatorname{ad}_{-1}^{\lambda}\right)^{k+1} x$ является ненулевым подпространством в $\mathfrak{f}_{-1}^{\lambda}$. Неприводимость $T_{\lambda}$ означает, что $\mathfrak{i}_{-1}=\mathfrak{f}_{-1}^{\lambda}$. В силу тождества Якоби $\left[\mathfrak{i}_{-1}, \mathfrak{f}_{1}^{\lambda}\right] \subset \mathfrak{i}_{0}$ есть идеал в $\mathfrak{f}_{0}^{\lambda}$. Но единственным нетривиальным идеалом в $\mathfrak{f}_{0}^{\lambda}=\mathfrak{a s}$ является центр. Значит, из включения $q_{i} \partial \xi_{i} \in$ $\left[\mathfrak{i}_{-1}, \mathfrak{h}_{1}\right]=\left[\mathfrak{h}_{-1}, \mathfrak{h}_{1}\right]$ следует равенство $\mathfrak{i}_{0}=\mathfrak{h}_{0}$. В частности, $\mathfrak{i}_{0}$ содержит элемент $T_{\lambda}(z)=-\lambda \sum\left(q_{i} \partial q_{i}+\xi_{i} \partial \xi_{i}\right)$. Но $\left[T_{\lambda}(z), h\right]=-\lambda k h$ для всех $h \in \mathfrak{f}_{k}^{\lambda}$. Следовательно, $\mathfrak{i}=\mathfrak{f}^{\lambda}$ и $\mathfrak{f}^{\lambda}$ проста.

Пункт 2) вытекает из изоморфизма неположительных частей.

Мы будем обозначать изоморфные простые супералгебры Ли $\mathfrak{f}^{\lambda}$ через $\mathfrak{v a s}(4 \mid 4)$.

ЗАМЕЧАНИЕ 2.2. Так каK as $\subset \mathfrak{s l}(4 \mid 4)$, то $\mathfrak{v a s}(4 \mid 4) \subset \mathfrak{s v e c t}(4 \mid 4)$.

Любое векторное поле $D \in \mathfrak{v e c t}(4 \mid 4)$ представляется в виде

$$
D=\sum_{i \leqslant 4}\left(P_{i} \partial \xi_{i}+Q_{i} \partial q_{i}\right), \quad \text { где } P_{i}, Q_{i} \in \mathbb{C}[q, \xi] \text {. }
$$

Тогда система уравнений, описанная в п. 1.5 и выделяющая $\mathfrak{f}^{\lambda}$ в vect $(4 \mid 4)$, будет иметь вид

$$
\begin{gathered}
\frac{\partial Q_{i}}{\partial q_{j}}+(-1)^{p(D)} \frac{\partial P_{j}}{\partial \xi_{i}}=0 \quad \text { для любых } i \neq j, \\
\frac{\partial Q_{i}}{\partial q_{i}}+(-1)^{p(D)} \frac{\partial P_{i}}{\partial \xi_{i}}=\frac{1}{2} \sum_{1 \leqslant j \leqslant 4} \frac{\partial Q_{j}}{\partial q_{j}} \quad \text { для } i=1,2,3,4,
\end{gathered}
$$




$$
\begin{gathered}
\frac{\partial Q_{i}}{\partial \xi_{j}}+\frac{\partial Q_{j}}{\partial \xi_{i}}=0 \quad \text { для любых } i, j, \\
\frac{\partial P_{i}}{\partial q_{j}}-\frac{\partial P_{j}}{\partial q_{i}}=(-1)^{p(D)} \lambda\left(\frac{\partial Q_{k}}{\partial \xi_{l}}-\frac{\partial Q_{l}}{\partial \xi_{k}}\right) \\
\text { для любой четной подстановки }\left(\begin{array}{cccc}
1 & 2 & 3 & 4 \\
i & j & k & l
\end{array}\right) .
\end{gathered}
$$

Система (2.3)-(2.6) стандартными методами может быть решена явно. В результате мы получаем следующую теорему.

Теорема 2.3. Любое векторное поле $D \in \mathfrak{f}^{\lambda}$ может быть представлено в виде

$$
D=D_{f}+c Z,
$$

где $c \in \mathbb{C}, Z=\sum_{i \leqslant 4}\left(q_{i} \partial q_{i}+\xi_{i} \partial \xi_{i}\right), f \in \mathfrak{s l} \mathfrak{e}^{\circ}(4) u$

$$
\begin{gathered}
D_{f}=\operatorname{Le}_{f}+\lambda\left(-\operatorname{Le}_{\hat{f}}+2 \sum_{1 \leqslant i \leqslant 4 ;(i, j, k, l) \in A_{4}} \frac{\partial^{3}\left(\Delta^{-1}(f)\right)}{\partial \xi_{j} \partial \xi_{k} \partial \xi_{l}} \partial \xi_{i}\right) \\
\hat{f}=4 \Delta^{-1}\left(\frac{\partial^{4}\left(\Delta^{-1}(f)\right)}{\partial \xi_{1} \partial \xi_{2} \partial \xi_{3} \partial \xi_{4}}\right)
\end{gathered}
$$

(через $A_{4} \subset S_{4}$ мы обозначаем подгруппу четных подстановок, а $\Delta^{-1}$ определен в $n .1 .2)$.

СлЕДСтвиЕ 2.4. 1) Супералгебра Ли $\mathfrak{f}^{\lambda}$ является деформачией супералгебры $\pi u \mathfrak{s l e}^{\circ}(4) \rightarrow \mathbb{C} Z$.

2) Если $\operatorname{deg}_{\xi} f \leqslant 1$, то $D_{f}=\operatorname{Le}_{f} u$, следовательно, подалгебра $\mathfrak{h}=\left\{c Z+D_{f}:\right.$ $\left.\operatorname{deg}_{\xi} f \leqslant 1, c \in \mathbb{C}\right\}$ остается жесткой относительно этой деформачии.

3) Пусть $\Omega=d q_{1} \wedge d q_{2} \wedge d q_{3} \wedge d q_{4}$ есть форма объема на подстилающем многообразии супермногообразия $\mathscr{C}^{4 \mid 4}$. Как мы уже отмечали, элемент объема $\operatorname{vol}(q, \xi)$ во всем суперпространстве $\mathscr{C}^{4 \mid 4}$ инвариантен относительно действия $\mathfrak{f}^{\lambda}$, чего нельзя сказать о форме $\Omega$. Однако она инвариантна относительно жесткой подалгебрьи $\mathfrak{h}$.

4) Пусть $D \in \mathfrak{f}^{\lambda}, L_{D}$ есть производная Ли вдоль $D, a \omega^{-1}-$ бивектор, двойственный $\kappa \omega$. Тогда уравнения (2.3)-(2.6) можно переписать в виде

$$
L_{D} \omega=\frac{1}{2}\left(\sum \frac{\partial Q_{i}}{\partial q_{i}}\right) \omega+\lambda \omega^{-1}\left(L_{D} \Omega\right)
$$

Заменив коэффичиент при $\omega$ в правой части формуль (2.7) на произвольную функиию $\Psi_{D}$, но добавив условие $\operatorname{div} D=0$, автоматически получим, что $\Psi(u, \xi)=\frac{1}{2} \sum \partial Q_{i} / \partial u_{i}$. Поэтому супералгебра Ли $\mathfrak{f}^{\lambda}$ может быть выделена системой уравнений

$$
\left\{\begin{aligned}
L_{D} \omega & =\Psi \omega+\lambda \omega^{-1}\left(L_{D} \Omega\right), \\
\operatorname{div} D & =0 .
\end{aligned}\right.
$$




\section{§3. Исключительная простая супералгебра Ли kas подалгебра в $\mathfrak{k}(1 \mid 6)$}

Реализуем контактную супералгебру Ли $\mathfrak{g}=\bigoplus_{i \geqslant-2} \mathfrak{g}_{i}=\mathfrak{k}(1 \mid 2 n)$ производящими функциями из $\mathbb{C}[t, \xi, \eta]$, где $\xi=\left(\xi_{1}, \ldots, \xi_{n}\right), \eta=\left(\eta_{1}, \ldots, \eta_{n}\right)$. Непосредственно проверяется, что компонента $\mathfrak{g}_{1}$ градуировки 1 порождает всю подалгебру $\mathfrak{g}_{+} \subset \mathfrak{g}$ элементов положительной градуировки. При этом сама $\mathfrak{g}_{1}$ как $\mathfrak{g}_{0}$-модуль является прямой суммой двух подмодулей $\mathfrak{g}_{\xi \eta}=\Lambda^{3}(\xi, \eta)$ и $\mathfrak{g}_{t}=t \Lambda^{1}(\xi, \eta)$.

Продолжения, порожденные этими подмодулями, хорошо известны:

$$
\begin{gathered}
\left(\mathfrak{g}_{-} \oplus \mathfrak{g}_{0}, \mathfrak{g}_{\xi \eta}\right)_{*}^{\mathfrak{m} \mathfrak{k}} \cong \mathfrak{p o}(0 \mid 2 n) \oplus \mathbb{C} K_{t} \cong \mathfrak{d} \mathfrak{e r}(\mathfrak{p o}(0 \mid 2 n)), \\
\left(\mathfrak{g}_{-} \oplus \mathfrak{g}_{0}, \mathfrak{g}_{t}\right)_{*}^{\mathfrak{m k}}=\mathfrak{g}_{-2} \oplus \mathfrak{g}_{-1} \oplus \mathfrak{g}_{0} \oplus \mathfrak{g}_{t} \oplus \mathbb{C} K_{t^{2}} \cong \mathfrak{o} \mathfrak{s p}(2 n \mid 2) .
\end{gathered}
$$

Заметим, что подмодуль $\mathfrak{g}_{t}$ всегда неприводим, а подмодуль $\mathfrak{g}_{\xi \eta}$ тривиален при $n=1$ и неприводим при всех $n>1$ и $n \neq 3$. И только в случае $\mathfrak{k}(1 \mid 6)$ он разлагается в сумму двух неприводимых подмодулей, которые мы обозначим $\mathfrak{g}_{\xi}$ и $\mathfrak{g}_{\eta}$. В этом случае $\mathfrak{g}_{0}=\mathfrak{o}(6) \cong \mathfrak{s l}(4)$. Как $\mathfrak{s l}(4)$-модули, $\mathfrak{g}_{\xi}$ и $\mathfrak{g}_{\eta}$ являются симметрическими квадратами $S^{2}(\mathrm{id})$ и $S^{2}\left(\mathrm{id}^{*}\right)$ тождественного 4-мерного представления и двойственного к нему соответственно. В реализации производящими функциями $\mathfrak{g}_{\xi}$ и $\mathfrak{g}_{\eta}$ получаются друг из друга заменой $\xi$ на $\eta$.

Теорема 3.1. 1) Картановское продолжение $\mathfrak{h}^{\xi}=\left(\mathfrak{g}_{-} \oplus \mathfrak{g}_{0}, \mathfrak{g}_{\xi} \oplus \mathfrak{g}_{t}\right)_{*}^{\mathfrak{m k}}$ бесконечномерно и просто. Оно изоморфно $\mathfrak{h}^{\eta}=\left(\mathfrak{g}_{-} \oplus \mathfrak{g}_{0}, \mathfrak{g}_{\eta} \oplus \mathfrak{g}_{t}\right)_{*}^{\mathfrak{m k}}$.

2) $\left(\mathfrak{g}_{-} \oplus \mathfrak{g}_{0}, \mathfrak{g}_{\xi}\right)_{*}^{\mathfrak{m k}} \cong\left(\mathfrak{g}_{-} \oplus \mathfrak{g}_{0}, \mathfrak{g}_{\eta}\right)_{*}^{\mathfrak{m k}} \cong \mathfrak{a s} \oplus \mathbb{C} K_{t} \cong \mathfrak{d e r}(\mathfrak{a s})$.

ДоказАтельство. Пункт 2) проверяется непосредственно; простота в п. 1) доказывается аналогично теореме 2.1. Чтобы убедиться в бесконечномерности супералгебры Ли $\mathfrak{h}^{\xi}$, заметим, что $K_{t^{2}} \in \mathfrak{h}^{\xi}$ и

$$
\text { ad } K_{t^{2}}=2 t(t \partial t+E-2), \quad \text { где } E=\sum\left(\xi_{i} \frac{\partial}{\partial \xi_{i}}+\eta_{i} \frac{\partial}{\partial \eta_{i}}\right) .
$$

Следовательно, $\operatorname{Ker}\left(\operatorname{ad} K_{t^{2}}\right)$ в $\mathfrak{k}(1 \mid 6)$ состоит из полей с такими производящими функциями $f$, что $\operatorname{deg}_{t} f+\operatorname{deg}_{\xi} f+\operatorname{deg}_{\eta} f-2=0$, т. е. $\operatorname{Ker}\left(\operatorname{ad} K_{t^{2}}\right) \cong \mathfrak{s l}(4) \oplus \mathfrak{g}_{t} \oplus$ $\mathbb{C} K_{t^{2}}$. Значит, ad $K_{t^{2}}$ задает вложения $\mathfrak{s l}(4)$-модулей: $\mathfrak{g}_{\xi}$ в $\mathfrak{h}_{3}^{\xi}$ и $\mathfrak{h}_{k}^{\xi}$ в $\mathfrak{h}_{k+2}^{\xi}$ при $k>1$.

Мы обозначаем простую исключительную супералгебру Ли, описанную в п. 1) теоремы 3.1 , через kas.

ЗАмЕЧАния 3.2.1) Поскольку при $k>4$ производящие функции всех полей из $\mathfrak{k}(1 \mid 6)_{k}$ делятся на $t$, из (3.2) следует, что при $k>2$ оператор ad $K_{t^{2}}$ задает изоморфизм компонент $\mathfrak{k}(1 \mid 6)_{k}$ и $\mathfrak{k}(1 \mid 6)_{k+2}$. Непосредственно проверяется, что $\mathfrak{h}_{k}^{\xi} \oplus \mathfrak{h}_{k}^{\eta}=\mathfrak{k}(1 \mid 6)_{k}$ при $k=3,4$. Действие ad $K_{t^{2}}$ продолжает это равенство на все $k \geqslant 3$. Таким образом, $\mathfrak{k}(1 \mid 6)=\mathfrak{h}^{\xi}+\mathfrak{h}^{\eta}$ и в силу $(3.1) \mathfrak{h}^{\xi} \cap \mathfrak{h}^{\eta}=\mathfrak{o s p}(6 \mid 2)$.

2) Как $\mathfrak{s l}(4)$-модули компоненты $\mathfrak{k a s}_{k}$ при $k \geqslant 0$ имеют вид $\mathfrak{k a s}_{2 k}=\operatorname{ad} \oplus \mathbb{C}$, $\mathfrak{k a s}_{2 k+1}=S^{2}(\mathrm{id}) \oplus \Lambda^{2}(\mathrm{id})$.

3) Компонента $\mathfrak{k a s}_{1}$ порождает всю подалгебру $\bigoplus_{k \geqslant 1} \mathfrak{k a s}_{k}$ компонент положительной градуировки. 


\section{§4. Исключительное картановское продолжение $\mathfrak{v l e}(4 \mid 3)$}

В этом и следующем параграфах мы используем обозначение сқ для тривиального центрального расширения супералгебры Ли $\mathfrak{g}$ и $\mathfrak{d g}$ для супералгебры Ли, получающейся из $\mathfrak{g}$ добавлением некоторого оператора внешнего дифференцирования.

Пусть $\mathfrak{u}=\mathfrak{v e c t}(m \mid n)$, а $\mathfrak{g}=\left(\mathfrak{u}_{-1}, \mathfrak{g}_{0}\right)_{*}-$ простая подсупералгебра Ли в $\mathfrak{u}$. Пусть, кроме того, существует элемент $D \in \mathfrak{u}_{0}$, задающий внешнее дифференцирование на $\mathfrak{g}$ и не имеющий ядра на $\mathfrak{u}_{+}=\bigoplus_{i>0} \mathfrak{u}_{i}$. Нас будет интересовать продолжение $\tilde{\mathfrak{g}}=\left(\mathfrak{g}_{-1}, \mathfrak{g}_{0} \oplus \mathbb{C} D\right)_{*}$.

ЛЕмма 4.1. 1) Либо продолжение $\tilde{\mathfrak{g}}$ просто, либо $\tilde{\mathfrak{g}}=\mathfrak{g} \oplus \mathbb{C} D$.

2) Если $\tilde{\mathfrak{g}}$ просто, то для любого $v \in \mathfrak{g}_{-1}$ найдется такой элемент $F \in \tilde{\mathfrak{g}}_{1}$, uто $[v, F]=D+g$, где $g \in \mathfrak{g}_{0}$.

ДокАЗАТЕльство. Доказательство п. 1) аналогично доказательству теоремы 2.1. Для доказательства п. 2) достаточно заметить, что отображение $F \mapsto \alpha_{F} \in \mathfrak{g}_{-1}^{*}$, определенное соотношением $[v, F]=\alpha_{F}(v) D+g$, где $g \in \mathfrak{g}_{0}$, $v \in \mathfrak{g}_{-1}, F \in \tilde{\mathfrak{g}}_{1}$, задает гомоморфизм $\mathfrak{g}_{0}$-модулей.

Пусть $\mathfrak{u}=\mathfrak{v e c t}\left(2^{n-1} \mid 2^{n-1}-1\right)$. Реализуем $\mathfrak{u}_{-1}$ как $\Pi\left(\Lambda\left(\xi_{1}, \ldots, \xi_{n}\right) / \mathbb{C} \cdot 1\right)$. Как мы отмечали в п. 1.5 , простая супералгебра Ли $\mathfrak{l}(n ; n)$ является картановским продолжением $\left(\Pi\left(\Lambda\left(\xi_{1}, \ldots, \xi_{n}\right) / \mathbb{C} \cdot 1\right), \mathfrak{v e c t}(0 \mid n)\right)_{*}$. В качестве $D$ возьмем градуирующий оператор в $\mathfrak{u}$. Тогда $\mathfrak{g}_{0} \oplus \mathbb{C} D \cong \mathfrak{c v e c t}(0 \mid n)$.

ПримеР 4.2. При $n=2$ имеем $\operatorname{dim} \mathfrak{g}_{-1}=2 \mid 1, \mathfrak{g}_{0}=\operatorname{vect}(0 \mid 2) \cong \mathfrak{s l}(2 \mid 1)$ и $\left(\mathfrak{g}_{-1}, \mathfrak{g}_{0}\right)_{*}=\mathfrak{l e}(2 ; 2) \cong \mathfrak{s v e c t}(2 \mid 1)$. Тогда $\mathfrak{c}\left(\mathfrak{g}_{0}\right)=\mathfrak{g l l}(2 \mid 1)$ и $\tilde{\mathfrak{g}}=\mathfrak{v e c t}(2 \mid 1)$.

ТеОРема 4.3. 1) Продолжение $\tilde{\mathfrak{g}}=(\Pi(\Lambda(3) / \mathbb{C} \cdot 1), \mathfrak{c v e c t}(0 \mid 3))_{*}$ является простой бесконечномерной подсупералгеброй в $\mathfrak{v e c t}(4 \mid 3)$.

2) $\tilde{\mathfrak{g}}=\left(\Pi\left(\Lambda\left(\xi_{1}, \ldots, \xi_{n}\right) / \mathbb{C} \cdot 1\right), \mathfrak{c} \mathfrak{v e c t}(0 \mid n)\right)_{*} \cong \mathfrak{d}(\mathfrak{l e}(n ; n))$ nрu $n>3$.

ДокаЗАтельство. Пункт 1) в силу п. 1) леммы 4.1 следует из явного описания векторных полей из $\tilde{\mathfrak{g}}$, которое мы приведем в теореме 4.4. Пункт 2) докажем от противного. Пусть $\tilde{\mathfrak{g}}$ проста. Возьмем $v=\xi_{1} \ldots \xi_{n}$ и $F_{v}$, как в п. 2) леммы 4.1. Тогда $\left[F, v_{1}\right](v)=(-1)^{p(v) p\left(v_{1}\right)}[F, v]\left(v_{1}\right)=-v_{1}+g v_{1}$ для любого $v_{1} \in \mathfrak{g}_{-1}$ и некоторого $g \in \mathfrak{g}_{0}$. В рассматриваемом $\tilde{\mathfrak{g}}_{0}$-модуле $\mathfrak{g}_{-1}$ элемент $v$ может отображаться только в функции степени $\geqslant n-1$. Следовательно, для всех функций $v_{1} \in \mathfrak{g}_{-1}$ степени $<n-1$ имеем $g v_{1}=v_{1}+\varphi\left(v_{1}\right)$, где $\operatorname{deg} \varphi\left(v_{1}\right) \geqslant n-1$. Но если $n-2 \geqslant 2$, то таких операторов в $\mathfrak{g}_{0}=\mathfrak{v e c t}(0 \mid n)$ просто нет. Полученное противоречие означает, что при $n>3$ супералгебра Ли $\tilde{\mathfrak{g}}$ не проста.

Исключительную простую супералгебру Ли $(\Pi(\Lambda(3) / \mathbb{C} \cdot 1), \mathfrak{c v e c t}(0 \mid 3))_{*}$ мы будем обозначать через $\mathfrak{v l e}(4 \mid 3)$. Опишем ее более подробно. Переобозначим базис в $\mathfrak{v l e}(4 \mid 3)_{-1}=\Pi\left(\Lambda\left(\eta_{1}, \eta_{2}, \eta_{3}\right)\right)$, полагая

$$
\Pi\left(\xi_{1} \xi_{2} \xi_{3}\right) \mapsto-\partial y, \quad \Pi\left(\xi_{i}\right) \mapsto-\partial q_{i}, \quad \Pi\left(\frac{\partial \xi_{1} \xi_{2} \xi_{3}}{\partial \xi_{i}}\right) \mapsto-\partial \xi_{i} .
$$

Каждый элемент $D \in \mathfrak{v e c t}(4 \mid 3)$ имеет вид $D=\sum_{i \leqslant 3}\left(P_{i} \partial \xi_{i}+Q_{i} \partial q_{i}\right)+Y \partial y$, где $P_{i}, Q_{i}, Y \in \mathbb{C}[y, q, \xi]$. В соответствии с п. 1.5 картановское продолжение $\mathfrak{v} \mathfrak{e}(4 \mid 3)$ может быть выделено в $\mathfrak{v e c t}(4 \mid 3)$ системой линейных дифференциальных уравнений. 
ТЕОРема 4.4. 1) Однородное (относительно четности) векторное поле $D \in \mathfrak{v e c t}(4 \mid 3)$ принадлежит $\mathfrak{v l e}(4 \mid 3)$ тогда и только тогда, когда оно удовлетворяет следующей системе уравнений:

$$
\begin{aligned}
& \frac{\partial Q_{i}}{\partial q_{j}}+(-1)^{p(D)} \frac{\partial P_{j}}{\partial \xi_{i}}=0 \quad \text { для любьих } i \neq j, \\
& \frac{\partial Q_{i}}{\partial q_{i}}+(-1)^{p(D)} \frac{\partial P_{i}}{\partial \xi_{i}}=\frac{1}{2}\left(\sum_{1 \leqslant j \leqslant 3} \frac{\partial Q_{j}}{\partial q_{j}}+\frac{\partial Y}{\partial y}\right) \quad \partial л \Omega \quad i=1,2,3, \\
& \frac{\partial Q_{i}}{\partial \xi_{j}}+\frac{\partial Q_{j}}{\partial \xi_{i}}=0 \text { для любых } i, j, \text { в частности, } \frac{\partial Q_{i}}{\partial \xi_{i}}=0, \\
& \frac{\partial P_{i}}{\partial q_{j}}-\frac{\partial P_{j}}{\partial q_{i}}=-(-1)^{p(D)} \frac{\partial Y}{\partial \xi_{k}} \\
& \text { для любой четной подстановки }\left(\begin{array}{lll}
1 & 2 & 3 \\
i & j & k
\end{array}\right) \text {, } \\
& \frac{\partial Q_{i}}{\partial y}=0 \quad \text { для } i=1,2,3 \\
& \frac{\partial P_{k}}{\partial y}=(-1)^{p(D)} \frac{1}{2}\left(\frac{\partial Q_{i}}{\partial \xi_{j}}-\frac{\partial Q_{j}}{\partial \xi_{i}}\right) \\
& \text { для любой четной подстановки }\left(\begin{array}{lll}
1 & 2 & 3 \\
i & j & k
\end{array}\right) \text {. }
\end{aligned}
$$

2) Каждое решение системь (4.1)-(4.6) имеет вид

$$
\begin{aligned}
D=\operatorname{Le}_{f}+ & y B_{f}-(-1)^{p(f)}\left(y \Delta(f)+y^{2} \frac{\partial^{3} f}{\partial \xi_{1} \partial \xi_{2} \partial \xi_{3}}\right) \partial y \\
& +B_{g}-(-1)^{p(g)}\left(\Delta(g)+2 y \frac{\partial^{3} g}{\partial \xi_{1} \partial \xi_{2} \partial \xi_{3}}\right) \partial y,
\end{aligned}
$$

где $f, g \in \mathbb{C}[q, \xi]$ и оператор $B_{f}$ задается формулой

$$
B_{f}=\frac{\partial^{2} f}{\partial \xi_{2} \partial \xi_{3}} \frac{\partial}{\partial \xi_{1}}+\frac{\partial^{2} f}{\partial \xi_{3} \partial \xi_{1}} \frac{\partial}{\partial \xi_{2}}+\frac{\partial^{2} f}{\partial \xi_{1} \partial \xi_{2}} \frac{\partial}{\partial \xi_{3}} .
$$

Доказательство чисто техническое и совершенно стандартное.

Заметим, что по построению у нас есть вложение $i_{1}: \mathfrak{l e}(3 ; 3) \rightarrow \mathfrak{v l e}(4 \mid 3)$, coхраняющее градуировку супералгебры $\mathfrak{k}(3 ; 3)$. Формулы $(4.7),(4.8)$ позволяют описать его явно:

$$
i_{1}\left(\operatorname{Le}_{f(q)}\right)=\operatorname{Le}_{\sum}\left(\partial f / \partial q_{i}\right) \xi_{j} \xi_{k}-y f
$$

где $y$ рассматривается как параметр, а $(i, j, k)$ - четная подстановка,

$$
i_{1}\left(\operatorname{Le}_{\sum f_{i}(q) \xi_{i}}\right)=\operatorname{Le}_{f}-\varphi(q) \sum \xi_{i} \partial \xi_{i}+\left(-\varphi(q) y+\Delta\left(\varphi(q) \xi_{1} \xi_{2} \xi_{3}\right)\right) \partial y,
$$

где $\varphi(q)=\Delta(f)$,

$$
i_{1}\left(\operatorname{Le}_{\sum_{1 \leqslant i \leqslant 3,(i, j, k) \in A_{3}}} \psi_{i}(q) \xi_{k} \xi_{l}\right)=B_{f}-\Delta(f) \partial y, \quad i_{1}\left(\operatorname{Le}_{\psi(q) \xi_{1} \xi_{2} \xi_{3}}\right)=-\psi(q) \partial y .
$$

Кроме того, из $(4.7),(4.8)$ видно, что у нас есть и второе вложение $i_{2}: \mathfrak{l e}(3) \rightarrow$ $\mathfrak{v} \mathfrak{l}(4 \mid 3)$, сохраняющее градуировку супералгебры $\mathfrak{l}(3)$ :

$$
i_{2}\left(\operatorname{Le}_{f}\right)=\operatorname{Le}_{f}+y B_{f}-(-1)^{p(f)}\left(y \Delta(f)+y^{2} \frac{\partial^{3} f}{\partial \xi_{1} \partial \xi_{2} \partial \xi_{3}}\right) \partial y .
$$


При этом $i_{1}(\mathfrak{l e}(3 ; 3))+i_{2}(\mathfrak{l} \mathfrak{e}(3))=\mathfrak{v} \mathfrak{k}(4 \mid 3)$, а $i_{1}(\mathfrak{l} \mathfrak{e}(3 ; 3)) \cap i_{2}(\mathfrak{l} \mathfrak{e}(3))=\mathfrak{s l} \mathfrak{e}^{\circ}(3 ; 3) \cong$ $\mathfrak{s l e}^{\circ}(3)$. Отображение $\varphi$, определяемое формулами

$$
\left.\varphi\right|_{i_{1}(\mathfrak{l} \mathfrak{e}(3 ; 3))}=\operatorname{sign} \circ i_{2} i_{1}^{-1},\left.\quad \varphi\right|_{i_{2}(\mathfrak{r}(3))}=i_{1} i_{2}^{-1},
$$

задает нетривиальный автоморфизм супералгебры Ли $\mathfrak{v} \mathfrak{e}(4 \mid 3)$, ограничение которого на $\mathfrak{s l e}^{\circ}(3)$ есть $R$ (см. п. 1.3). Из формул (4.7), (4.8) видно также, что этот автоморфизм может быть описан во внутренних терминах супералгебры Ли $\mathfrak{v e c t}(4 \mid 3)$ как переградуировка: $\operatorname{deg} y=-1, \operatorname{deg} \xi_{i}=0, \operatorname{deg} q_{i}=1$. Конечно, на всей супералгебре vect(4|3) эта переградуировка нарушает как условие конечномерности однородных компонент, так и условие конечности глубины. Однако на $\mathfrak{v} \mathfrak{l}(4 \mid 3)$ она удовлетворяет обоим этим условиям.

\section{$\S 5$. Исключительные простые супералгебры Ли глубины 2 $\mathfrak{m b}(4 \mid 5)$ и $\mathfrak{k s l e}(9 \mid 6)$}

5.1. Две последние простые исключительные супералгебры Ли строятся с помощью конструкции $\mathfrak{m k}$-продолжения.

Пусть $\mathfrak{u}=\bigoplus_{i \geqslant-2} \mathfrak{u}_{i}$ есть либо $\mathfrak{m}(n)$, либо $\mathfrak{k}(2 m+1 \mid n) ;$ пусть $\mathfrak{g}=\left(\mathfrak{u}_{-}, \mathfrak{g}_{0}\right)_{*}^{\mathfrak{m} \mathfrak{k}}$ - такая подалгебра в $\mathfrak{u}$, для которой подпространство $\mathfrak{u}_{-2}$ является центром в $\mathfrak{g}$, а факторалгебра $\mathfrak{g} / \mathfrak{u}_{-2}$ проста. Пусть, кроме того, $D \in \mathfrak{u}_{0}$ задает внешнее дифференцирование на $\mathfrak{g}$ и не имеет ядра на $\mathfrak{u}_{-2} \oplus \mathfrak{u}_{+}$, где $\mathfrak{u}_{+}=\bigoplus_{i>0} \mathfrak{u}_{i}$. Нас будет интересовать $\mathfrak{m k}$-продолжение $\tilde{\mathfrak{g}}=\left(\mathfrak{g}_{-}, \mathfrak{g}_{0} \oplus \mathbb{C} D\right)_{*}^{\mathfrak{m} \mathfrak{k}}$.

Лемма 5.1. Либо $\tilde{\mathfrak{g}}$ проста, либо $\tilde{\mathfrak{g}} \cong \mathfrak{d} \mathfrak{g}$

Эта лемма аналогична лемме 4.1 для картановского продолжения, и ее доказательство стандартно.

5.2. Описание $\mathfrak{m b}(4 \mid 5)$. Пусть $\hat{\mathfrak{g}}=\mathfrak{b}_{1 / 2}(n ; n)$ (см. п. 1.3). Тогда действие $\hat{\mathfrak{g}}_{0}$ на $\hat{\mathfrak{g}}_{-1}$ сохраняет невырожденную суперкососимметрическую форму

$$
\omega(\varphi \sqrt{\mathrm{vol}}, \psi \sqrt{\mathrm{vol}})=\int \varphi \psi \mathrm{vol}, \quad p(\omega) \equiv n \quad(\bmod 2) .
$$

Поэтому мы можем рассмотреть нетривиальное центральное расширение $\mathfrak{g}=$ $\tilde{\mathfrak{c}}\left(\mathfrak{b}_{1 / 2}(n ; n)\right)$ супералгебры Ли $\hat{\mathfrak{g}}$ глубины 2 , задаваемое с помощью формы $\omega$. Это центральное расширение естественным образом вкладывается в

$$
\mathfrak{u}= \begin{cases}\mathfrak{m}\left(2^{n-1}\right) & \text { для нечетных } n, \\ \mathfrak{k}\left(1+2^{n-1} \mid 2^{n-1}\right) & \text { для четных } n .\end{cases}
$$

В качестве оператора $D$, участвующего в лемме 5.1, возьмем градуирующий оператор из $\mathfrak{u}_{0}$, т.е. $\mathfrak{g}_{0} \oplus \mathbb{C} D \cong \mathfrak{c}\left(\mathfrak{g}_{0}\right)$.

ПримеР 5.2. Пусть $n=2$. Тогда $\mathfrak{g}_{-}=\mathfrak{h} \mathfrak{e i}(2 \mid 2)$ и $\mathfrak{c}\left(\mathfrak{g}_{0}\right)=\mathfrak{c v e c t}(0 \mid 2) \cong \mathfrak{c o s p}(2 \mid 2)$, $\mathrm{a}(\mathfrak{h e i}(2 \mid 2), \mathfrak{c v e c t}(0 \mid 2))_{*} \cong \mathfrak{k}(3 \mid 2)$. Положим

$$
\mathfrak{g}_{-}= \begin{cases}\mathfrak{a} \mathfrak{b}\left(2^{n-1}\right) & \text { для нечетных } n, \\ \mathfrak{h e i}\left(2^{n-1} \mid 2^{n-1}\right) & \text { для четных } n .\end{cases}
$$

ТЕОРема 5.3. 1) $(\mathfrak{a} \mathfrak{b}(4), \mathfrak{c v e c t}(0 \mid 3))_{*}^{\mathfrak{m k}}$ являетсл простой супералгеброй Ли.

2) $\left(\mathfrak{g}_{-}, \mathfrak{c} \mathfrak{v e c t}(0 \mid n)\right)_{*}^{\mathfrak{m} \mathfrak{k}} \cong \mathfrak{d}\left(\mathfrak{g}_{-}, \mathfrak{v e c t}(0 \mid n)\right)_{*}^{\mathfrak{m k}} \partial \jmath_{\Omega} n>3$.

ДоКАЗАТЕЛЬСТво. В силу леммы 5.1 в п. 1) нам достаточно просто проверить, что в компоненте $\mathfrak{m}(4)_{1}$ есть элемент, все коммутаторы которого с $\mathfrak{m}(4)_{-1}$ 
попадают в $\mathfrak{c v e c t}(0 \mid 3) \subset \mathfrak{m}(4)_{0}$, но не все в $\mathfrak{v e c t}(0 \mid 3)$. Это проверяется непосредственным вычислением. Пункт 2) доказывается аналогично теореме 4.3.

Исключительную простую супералгебру Ли, указанную в п. 1) теоремы 5.3, мы будем обозначать $\mathfrak{m b}(4 \mid 5)$. Опишем ее строение. Для этого рассмотрим две супералгебры Ли, изоморфные (изоморфизм осуществляется с помощью $R$, см. п. 1.3) друг другу как абстрактные, но не как $\mathbb{Z}$-градуированные супералгебры, $\mathfrak{g}=\tilde{\mathfrak{c}}\left(\mathfrak{b}_{1 / 2}(3 ; 3)\right)$ и $\mathfrak{h}=\tilde{\mathfrak{c}}\left(\mathfrak{b}_{-3,1}(3)\right)$. По построению у нас есть вложение $i_{1}: \mathfrak{g} \rightarrow \mathfrak{m b}(4 \mid 5)$, сохраняющее $\mathbb{Z}$-градуировку супералгебры $\mathfrak{g}$. Кроме того, $\mathfrak{g} \supset \mathfrak{e s l e}^{\circ}(3 ; 3)$ (cм. п. 1.4). Тогда $i_{2}=\left.i_{1} R\right|_{\mathfrak{e s l e}}{ }^{\circ}(3 ; 3)$ задает вложение, сохраняющее градуировку в $\mathfrak{e s l e}^{\circ}(3)$. Неположительные части супералгебр $\mathfrak{h}$ и $\mathfrak{e s l e}^{\circ}(3)$ отличаются лишь одним элементом $z=M_{-\sum} q_{i} \xi_{i}-3 \tau$. Непосредственным вычислением проверяется, что действие $z$ на $\mathfrak{h}_{-1}$ совпадает с действием элемента $-3 D-$ $\frac{1}{2} i_{1} R(z)$ на $i_{2}\left(\mathfrak{h}_{-1}\right)$. Таким образом, вложение $i_{2}$ продолжается до вложения неположительной части супералгебры $\mathfrak{h}$, а в силу универсальности mk-продолжения и до вложения всей $\mathfrak{h}$. При этом $i_{1}\left(\tilde{\mathfrak{c}}\left(\mathfrak{b}_{1 / 2}(3 ; 3)\right)\right)+i_{2}(\tilde{\mathfrak{c}}(\mathfrak{b}-3,1(3)))=\mathfrak{m} \mathfrak{b}(4 \mid 5)$, a $i_{1}\left(\tilde{\mathfrak{c}}\left(\mathfrak{b}_{-1 / 2}(3 ; 3)\right)\right) \cap i_{2}\left(\tilde{\mathfrak{c}}\left(\mathfrak{b}_{-3,1}(3)\right)\right)=\mathfrak{e s l e}^{\circ}(3)$. Отображение $\varphi$, такое, что

$$
\left.\varphi\right|_{i_{1}\left(\tilde{\mathfrak{c}}\left(\mathfrak{b}_{1 / 2}(3 ; 3)\right)\right)}=\operatorname{sign} \circ i_{2} i_{1}^{-1},\left.\quad \varphi\right|_{i_{2}\left(\tilde{\mathfrak{c}}\left(\mathfrak{b}_{-3,1}(3)\right)\right)}=i_{1} i_{2}^{-1}
$$

задает нетривиальный автоморфизм супералгебры $\mathfrak{m b}(4 \mid 5)$, ограничение которого на $\mathfrak{e s l e}^{\circ}(3)$ есть $R$.

Введем координаты $u_{0}, u_{1}, u_{2}, u_{3}, \eta_{0}, \eta_{1}, \eta_{2}, \eta_{3}$ на $\mathfrak{m}(4)$ (в реализации производящими функциями) по формулам $u_{0}=i_{1}(1), \eta_{0}=i_{1}\left(\xi_{1} \xi_{2} \xi_{3}\right), \eta_{k}=$ $i_{1}\left(\xi_{k}\right), u_{k}=i_{1}\left(\partial\left(\xi_{1} \xi_{2} \xi_{3}\right) / \partial \xi_{k}\right)$ при $k>0$. Тогда автоморфизм $\varphi$ может быть описан во внутренних терминах алгебры $\mathfrak{m}(4)$ как переградуировка: $\operatorname{gr} f=$ $\operatorname{deg} f-1$ и $\operatorname{deg} u_{0}=-1, \operatorname{deg} \eta_{k}=0, \operatorname{deg} u_{k}=\operatorname{deg} \tau=1, \operatorname{deg} \eta_{0}=2$. Как и в случае $\mathfrak{v} \mathfrak{l e}(4 \mid 3)$, эта переградуировка действует очень плохо на всей алгебре $\mathfrak{m}(4)$.

5.3. Описание $\mathfrak{k} \mathfrak{k}(\mathbf{e} \mid \mathbf{6})$. Определим нетривиальное центральное расширение $\mathfrak{g}=\tilde{\mathfrak{c}}\left(\mathfrak{s l e}^{\circ}(n ; n)\right)$ глубины 2 супералгебры Ли $\mathfrak{s l e}^{\circ}(n ; n)$. Имеем $\mathfrak{g}_{0}=\mathfrak{s v e c t}(0 \mid n)$, $\mathfrak{g}_{-1}=\Pi(\tilde{\Lambda}(n) / \mathbb{C} \cdot 1)$. Центральное расширение строится с помощью суперкососимметрической $\mathfrak{g}_{0}$-инвариантной формы $\omega$, определенной на $\mathfrak{g}_{-1}$ :

Тогда

$$
\omega(f, g)=\int f g \operatorname{vol}(\xi) .
$$

$$
\mathfrak{g}_{-}= \begin{cases}\mathfrak{a} \mathfrak{b}\left(2^{n-1}-1\right) & \text { для нечетных } n, \\ \mathfrak{h} \mathfrak{e}\left(2^{n-1} \mid 2^{n-1}-2\right) & \text { для четных } n .\end{cases}
$$

Это означает, что $\mathfrak{m k}$-продолжение $\left(\mathfrak{g}_{-1}, \mathfrak{g}_{0}\right)_{*}^{\mathfrak{m k}}$ может быть вложено в $\mathfrak{u}=$ $\mathfrak{m}\left(2^{n-1}-1\right)$ при нечетных $n$ и в $\mathfrak{u}=\mathfrak{k}\left(1+2^{n-1} \mid 2^{n-1}-2\right)$ при четных $n$.

Пусть $x=\sum \xi_{i} \partial \xi_{i}$ - оператор стандартной $\mathbb{Z}$-градуировки в $\mathfrak{s v e c t ~}(0 \mid n)$, а $z=M_{\tau}$ или $z=K_{t}$ - оператор $\mathbb{Z}$-градуировки в $и$. Тогда для любых $a, b \in \mathbb{C}$ элемент $a x+b z$ задает внешнее дифференцирование $\mathfrak{g}_{0}$. Положим $\mathfrak{s v e c t}_{a, b}(n)=\mathfrak{s v e c t}(0 \mid n) \oplus \mathbb{C}(a x+b z)$. Нас будет интересовать $\mathfrak{k} \mathfrak{k}$-продолжение $\left(\mathfrak{g}_{-}, \mathfrak{s v e c t} \mathfrak{c}_{a, b}(n)\right)_{*}^{\mathfrak{m} \mathfrak{k}}$.

ПримеР 5.4. Пусть $n=3$. Тогда $\mathfrak{g}_{-1}=\Pi\left(\xi_{1}, \xi_{2}, \xi_{3}, \xi_{1} \xi_{2}, \xi_{1} \xi_{3}, \xi_{2} \xi_{3}\right)$ и $\mathfrak{g}_{-} \cong$ $\left(\mathfrak{b}_{\lambda}(3)\right)_{-}=\mathfrak{a b}(3)$ для любых $a, b$, a $\mathfrak{g}_{0}=\mathfrak{s v e c t}(0 \mid 3) \cong \mathfrak{s p e}(3)$. Поэтому $\mathfrak{g}_{0} \oslash \mathbb{C}(a x+$ $b z) \cong\left(\mathfrak{b}_{\lambda}(3)\right)_{0}$ для $\lambda=-b / 3 a$ и $\left(\mathfrak{a b}(3), \mathfrak{s v e c t}_{a, b}(3)\right)_{*}^{\mathfrak{m k}} \cong \mathfrak{b}_{\lambda}(3)$. В частности,

$$
\left(\mathfrak{a b}(3), \mathfrak{s v e c t}_{1,3}(3)\right)_{*}^{\mathfrak{m k}} \cong \mathfrak{s m}(3) \quad \text { и } \quad\left(\mathfrak{a b}(3), \mathfrak{s v e c t}_{1,0}(3)\right)_{*}^{\mathfrak{m k}} \cong \mathfrak{b}(3) .
$$


ТЕОРемА 5.5.1) $\left(\mathfrak{h e i}(8 \mid 6), \mathfrak{s v e c t}_{3,4}(4)\right)_{*}^{\mathfrak{m k}}$ является простой супералгеброй Ли

2) При $n>4$, а также при $(a, b) \notin \mathbb{C}(3,4)$ и $n=4$ имеем изоморфизм $\left(\mathfrak{g}_{-}, \mathfrak{s v e c t}_{a, b}(n)\right)_{*}^{\mathfrak{m k}} \cong \mathfrak{d}\left(\mathfrak{g}_{-}, \mathfrak{s v e c t}(0 \mid n)\right)_{*}^{\mathfrak{m} \mathfrak{k}}$.

ДокАЗАТЕЛЬСтво. Пункт 1) получается с помощью леммы 5.1 прямыми вычислениями. Пункт 2) доказывается аналогично теореме 4.3.

Мы будем обозначать простую исключительную супералгебру Ли из п. 1) через $\mathfrak{k} \mathfrak{s l e}(9 \mid 6)$.

Для описания ее строения заметим, что у нас уже есть вложение $i_{1}: \mathfrak{g}=$ $\tilde{\mathfrak{c}}\left(\mathfrak{s} \mathfrak{e}^{\circ}(n ; n)\right) \rightarrow \mathfrak{k} \mathfrak{s l e}(9 \mid 6)$. Кроме того, $\mathfrak{g} \supset \mathfrak{a s}$, причем это вложение сохраняет $\mathbb{Z}$-градуировку $(2.1)$ :

$$
\begin{gathered}
\mathfrak{a} \mathfrak{s}_{-2}=\mathfrak{g}_{-2}, \quad \mathfrak{a s}_{-1}=\left(\mathfrak{g}_{-1}\right)_{\overline{1}}=\Pi\left(\Lambda^{2}\left(\xi_{1}, \xi_{2}, \xi_{3}, \xi_{4}\right)\right), \\
\mathfrak{a s}_{0}=\mathfrak{s l}(4) \subset \mathfrak{g}_{0}=\mathfrak{s v e c t}(0 \mid 4), \quad \mathfrak{a s}_{1}=\Pi\left(S^{2}\left(q_{1}, q_{2}, q_{3}, q_{4}\right)\right) \subset \mathfrak{g}_{1} .
\end{gathered}
$$

Из теоремы 3.1 следует, что $\mathfrak{a s} \subset \mathfrak{k a s}$, причем неположительные части этих супералгебр отличаются друг от друга лишь одним элементом $K_{t}$. Переводя $K_{t}$ в элемент $-(3 x+4 z) / 2$, получим вложение неположительной части супералгебры kas в $\mathfrak{k} \mathfrak{s} \mathfrak{e}(9 \mid 6)$, продолжающееся до вложения $i_{2}: \mathfrak{k} \mathfrak{s} \rightarrow \mathfrak{k} \mathfrak{s l e}(9 \mid 6)$. При этом $i_{1}\left(\tilde{\mathfrak{c}}\left(\mathfrak{s l e}{ }^{\circ}(4 ; 4)\right)\right) \cap i_{2}(\mathfrak{k} \mathfrak{s})=\mathfrak{a s}$. Однако $i_{1}\left(\tilde{\mathfrak{c}}\left(\mathfrak{s l e}{ }^{\circ}(4 ; 4)\right)\right)+i_{2}(\mathfrak{k} \mathfrak{s}) \neq \mathfrak{k} \mathfrak{s} \mathfrak{l}(9 \mid 6)$.

\section{ЛиТЕРАТУРА}

1. Алексеевский Д. В., Лейтес Д. А., Щепочкина И. М. Примеры простых бесконечномерных супералгебр Ли векторных полей. C. R. Acad. Bulgare Sci., 34, №9, 1187-1190 (1980).

2. Grozman P., Leites D., Shchepochkina I. Lie superalgebras of string theories. Preprint hep-th 9702121.

3. Kac V. G. Lie superalgebras. Adv. Math., 26, 8-96 (1977).

4. Kotchetkoff Yu. Déformations de superalgébres de Buttin et quantification. C. R. Acad. Sci. Paris Sér. I, 299, No. 14, 643-645 (1984).

5. Кочетков Ю. Ю. Деформации супералгебр Ли. Деп. ВИНИТИ, М., 384-85 (1985).

6. Лейтес Д. А. Новые супералгебры Ли и механика. ДАН СССР , 18, № 5, 1277-1280 (1977).

7. Лейтес Д. А. Супералгебры Ли. В кн.: Современные проблемы математики, т. 25, ВИНИТИ, М., 1984, с. 3-49.

8. Leites D. Quantization. Supplement 3. In: Berezin F., Shubin M. Schrödinger equation. Kluwer, Dordrecht, 1991, pp. 483-522.

9. Post G., Shchepochkina I. Explicit bracket in an exceptional simple Lie superalgebra. Принято в Internat. J. Algebra Comput. (physics 9703022).

10. Щепочкина И. М. Исключительные простые бесконечномерные супералгебры Ли. C. R. Bulgare Sci., 36, No. 3, 313-314 (1983).

11. Shchepochkina I. Maximal subalgebras of simple Lie superalgebras. In: Seminar on Supermanifolds (Leites D., ed.), No. 32, Reports of Stockholm University, 1992, pp. 143 (hep-th 9702120).

12. Стернберг C. Лекции по дифференциальной геометрии. Мир, М., 1970. 